\title{
Intravitreal Fluocinolone Acetonide Implant and the Management of Non-infectious Posterior Uveitis
}

a report by

\author{
Carlos E Pavésio
}

Consultant Ophthalmic Surgeon, Moorfields Eye Hospital, London

\section{DOI: 10.17925/EOR.2007.00.00.79}

The term uveitis encompasses a large number of conditions, infectious or not, that can produce intraocular inflammation. The incidence of uveitis is probably greater than previously suspected. ${ }^{1}$ The anterior form is by far the most common, but the posterior form is the one that may lead to significant visual impairment, mostly as a consequence of chronic macular oedema. Some diseases will be localised to the eye or have the eye as its most important manifestation, while others will be systemic with the eye being only part of a larger problem.

\section{Management - General Considerations}

While the management of anterior disease is easily delivered by topical therapy, the treatment of the posterior form requires the use of peri-ocular injections or the use of systemic therapy, since topical therapy does not penetrate the posterior segment well. Peri-ocular injections attempt to concentrate high levels of long-acting or depo steroids around the eye, but are confronted with technical variability and the scleral barrier for intraocular penetration of the drug. They may be effective in $50 \%$ of cases, but their effect will last for only a few months and they have to be repeated, which increases the risk of local complications such as ptosis, extra-ocular muscle fibrosis and globe injury. Systemic therapy using primarily oral steroids supported by sparing agents, mostly in the form of immunosuppressive drugs, also faces the problem of a barrier (blood-ocular barrier) and the systemic distribution of the drugs. Both of these forms have their problems, either because of insufficient intraocular levels of the therapeutic agent, with consequent poor control of the disease, or because of unacceptable side effects. Overcoming these barriers becomes essential for the adequate management of posterior uveitis, from the point of view of both adequate intraocular levels and systemic side effects. The use of intraocular injections of steroids (mostly triamcinolone acetonide) has recently become quite common, but the duration of the effect is limited to a few months and further injections are needed if control of inflammation is to be maintained. ${ }^{2}$ This also increases the risk of complications, especially endophthalmitis. The use of a slow-release device could overcome the limitations of the injections and a few devices have been tested, some of which are degradable (Posurdex ${ }^{\circledR}$, which uses dexamethasone) and others non-degradable, such as the Retisert, which contains fluocinolone acetonide. The development of the Retisert was based on experience with Vitrasert ${ }^{\circledR}$, an intravitreal sustained-release delivery system for ganciclovir approved by the US Food and Drug Administration (FDA) in 1996 and by the European Medicines Agency

Carlos E Pavésio has been a Consultant Ophthalmic Surgeon at Moorfields Eye Hospital in London since 1995. He is Vice President of the Society for Ocular Immuno-Infectiology in Europe. He is involved in clinical trials investigating management strategies for uveitic cystoid macular oedema, intraocular lens implants in uveitic patients and visual outcomes in patients affected by choriocapillaropaties.

E: carlos.pavesio@moorfields.nhs.uk
(EMEA) in 1997 for the treatment of AIDS-related cytomegalovirus (CMV) retinitis. Other implants under investigation include Medidur ${ }^{\circledR}$, which also contains fluocinolone acetonide, and SurModics I-vation ${ }^{\mathrm{TM}}$, which also uses triamcinolone acetonide, both tested for diabetic macular oedema.

Intravitreal Fluocinolone Acetonide Implant - Retisert The Retisert developed by Bausch \& Lomb and Control Delivery Systems is the first device approved by the FDA for the specific indication to treat noninfectious posterior uveitis. Pellets containing the drug are coated in polyvinyl alcohol and silicone laminate and fixed to a polyvinyl alcohol suture strut. Fluocinolone acetonide is a synthetic corticosteroid with a potency similar to the glucocorticoid dexamethasone. It has the advantage of being much less soluble than the glucocorticoid, which allows the release of the drug over a much longer period of time. It was also chosen because the drug is very potent and allowed the creation of a very small implantable device (see Figure 1). In pharmacokinetic studies, drug delivery was linear, with no drug peaks and troughs and drug release was projected for approximately 1,000 days. ${ }^{3}$

\section{Studies}

The initial studies in human subjects were carried out in seven eyes of five patients with the diagnosis of Behçet's syndrome or idiopathic panuveitis. ${ }^{4}$ These patients had a mean duration of uveitis of six years before implantation of the device. All eyes remained quiet for the duration of the follow-up period. No eye required topical or peri-ocular steroids and systemic therapy was significantly reduced. It was interesting to note that three of the four cases of bilateral disease had severe inflammation of the fellow eye during the same period of follow-up. Two of these eyes had implants inserted later with marked improvement in the control of the inflammation. These implants contained $2.1 \mathrm{mg}$ of the drug, and the devices were designed to release $2 \mu \mathrm{g}$ of fluocinolone acetonide per day for approximately three years. These favourable results led to a randomised trial using the $2.1 \mathrm{mg}$ or a $0.59 \mathrm{mg}$ device, ${ }^{25}$ designed to release the drug at a targeted initial release rate of $2 \mu \mathrm{g} /$ day or $0.6 \mu \mathrm{g} / \mathrm{day}$, respectively, over a 1,000-day period. ${ }^{5}$ The inclusion criteria were the same as in the previous study and 36 eyes of 32 patients were studied. The results, over a longer follow-up period, showed a significant improvement with no recurrences during the first two years following implantation of the device compared with 2.5 episodes per eye the year preceding implantation. There were only five recurrences for the entire group during the total follow-up period. There was a marked reduction in the need for topical, peri-ocular and systemic therapy for the eyes with the device.

The results were so encouraging that a randomised, prospective, multicentre study was carried out in the US (27 sites) and at one site in Singapore to establish the role of the fluocinolone implant in the treatment of uveitis. ${ }^{6}$ In this study, 278 eyes were randomised to receive a $0.59 \mathrm{mg}$ or a $2.1 \mathrm{mg}$ device. Unilateral cases were preferred, but in bilateral cases the more 
Figure 1: Fluocinole Acetonide Implant

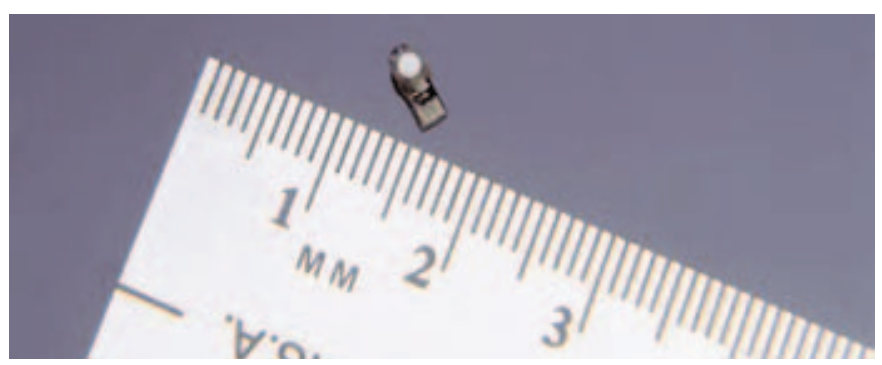

Figure 2: Retisert as Seen Inside the Eye after Implantation

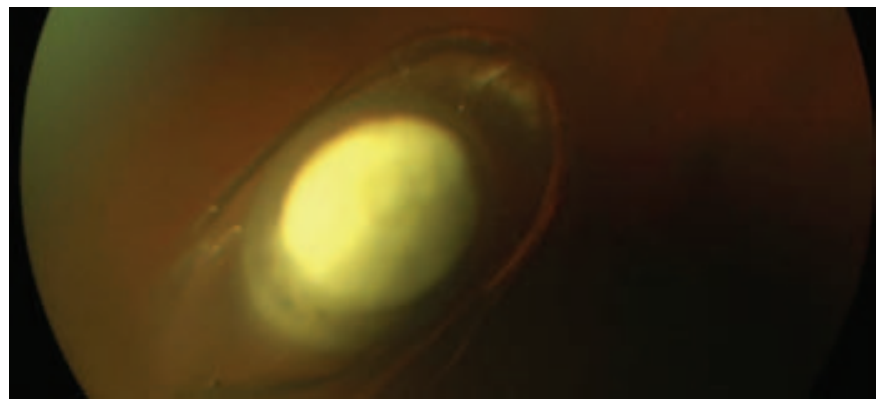

severely affected eye received the implant. The interim analysis at 34 weeks showed that implanted eyes had a very low recurrence rate compared with the 34 weeks prior to implantation (6.1×51.4\%). There was no difference between the recurrence rates for the two implants used. Again, the need for topical or systemic therapy was significantly reduced for the implanted eyes. A second multicentre pivotal trial carried out in the US, Canada and Asia confirmed these results and led to the FDA approval of this device for the management of non-infectious posterior uveitis in April 2005. In Europe, the $0.59 \mathrm{mg}$ device was tested against Standard of Care (SOC) guidelines, which represented the usual therapy employed by the different investigators at their centres, but following a specific protocol of which drugs to use and how they should be tapered. The $0.59 \mathrm{mg}$ device was chosen because, as stated above, its was equal to that of the $2.1 \mathrm{mg}$ device in terms of recurrence rate. The final analysis of the results of this study is still not available, but the data (unpublished) indicate a positive result that reaches statistical significance in terms of recurrence rate, recurrence frequency and frequency change compared with a similar period prior to implantation. Even though visual acuity was an outcome, it is important to stress that in these studies it was not considered a primary outcome, and the most important parameter was the ability of the drug to reduce recurrences. Vision at the time of enrolment might already have been affected permanently and the most important aspect was to demonstrate that no further deterioration occurred. In fact, visual acuity was found to stabilise or improve in most cases. The importance of controlling recurrences is the reduction of the cumulative damage to the eye generated by repeated inflammatory attacks, which will lead to progressive visual loss. ${ }^{7}$ If recurrences can be stopped, vision may be preserved.

\section{Implantation Procedure}

The implantation of the device involves a small surgical procedure. An incision in the sclera of about $3.5 \mathrm{~mm}$ in length is created at 4.0 or
$3.5 \mathrm{~mm}$ from the limbus. The implant is inserted into the posterior segment and sutured to the sclera. The construction of the wound is critical in avoiding post-operative complications such as hypotony and infections. This procedure can be performed under local or general anaesthetic. The implant is easily seen behind the crystalline lens after the operation (see Figure 2), but this does not aid assessment of the amount of drug still available in the device.

\section{Complications}

There are two groups of potential complications to consider, one associated with the procedure itself and the other linked to the steroid nature of the drug used. Surgical problems include haemorrhage, retinal detachment, implantation of the device in the wrong place (under the choroid and not in the vitreous, which does not allow it to work) and infection (endopthalmitis). Another complication associated with the surgical procedure was ocular hypotony, which was mainly due to poor construction of the wound. Side effects of steroids in the eye are wellknown and mainly result in raised intraocular pressure and cataract. Eyes receiving the implant required more topical intraocular pressure-lowering drops at 34 weeks compared with baseline (50x14\%) and 6\% of the eyes required a filtering procedure. ${ }^{6}$ After longer follow-up this figure goes up, reaching levels of about $20 \%$ for filtration surgery (unpublished data). Cataract was a very common complication, with $10 \%$ of eyes requiring surgery at 34-week follow-up - the figure increased with longer followup - with the majority of implanted eyes requiring cataract surgery, especially after 18 months (unpublished data).

\section{Conclusions}

Considering the many difficulties confronted by the clinician in the management of posterior uveitis and the limited options previously available, the development of a technology that allows effective local therapy is welcome. Avoiding systemic therapy whenever possible is always a desirable approach as it spares patients the many undesirable side effects of this route of drug administration. One of the concerns for these patients is quality of life, which can be severely affected by systemic therapy. The prolonged effect over about two and half years is also a very important aspect, but it still means that in a chronic disease a few procedures will be necessary over many years. The complications associated with the procedure are likely to become less important as surgeons become more familiar with the technique, especially wound construction and suturing. The two most frequent complications related to steroid use, namely cataract and raised intraocular pressure, are actually quite common in uveitis and were probably only accelerated by this form of therapy. Both of them can be dealt with efficiently and should not represent a reason not to implant the device. As mentioned above, vision may be preserved if recurrences can be better controlled - a well-established benefit of the implant, which may make it a more attractive option before vision becomes irreversibly damaged. This device is unlikely to be the final word in this chapter of the management of non-infectious posterior uveitis, but the technology of drug delivery it uses is likely to become very useful for the delivery of other drugs, as has already been shown for cyclosporine, $^{8}$ and may extend to other options such as anti-vascular endothelial growth factor (VEGF) therapy. This is an important development and will undoubtedly influence future approaches in this field.
1. Gritz DC, Wong IG, Ophtha/mology, 2004;111(3):491-500.

2. Beer PM, Bakri SJ, Singh RJ, et al., Ophthalmology, 2003;110(4):681-6.

3. Jaffe GJ, Yang $\mathrm{CH}$, Guo H, et al., Ophthalmol Vis Sci, 2000;41:3569-75.
4. Jaffe GJ, Ben-Nun J, Guo H, et al., Ophthalmology, 2000;107:2024-33.

5. Jafee GJ, McCallum RM, Branchaud B, et al., Ophthalmology, 2005;112:1192-8.

6. Jafee GJ, Martin D, Callanan D, et al., Ophtha/mology,
2006;113(6):1020-27.

7. Nguyen $Q D$, Callanan $D$, Dugel $P$, et al., Retina, 2006;(Suppl.):1-16.

8. Pearson PA, Jafee GJ, Martin DF, et al., Arch Ophthalmol, 1996;114:311-17. 


\section{The Ahmed ${ }^{\mathrm{Tm}}$ Elaucoma Valve}

Leading the way in Advanced Claucoma Drainage Technology

\section{Flexible Plate ${ }^{\mathrm{TM}} \boldsymbol{\Theta}$ Human Allograft Tissue}

Human

Allograft Tissue

Processed Sclera

\& Pericardium

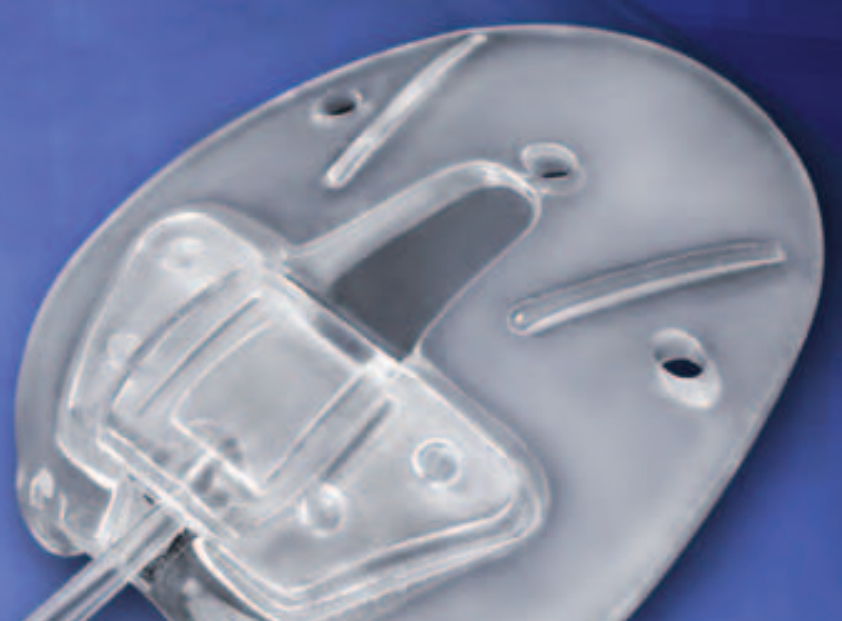

Model FP7 Flexible Plate ${ }^{\mathrm{m}}$ Surface area: $184.00 \mathrm{~mm}^{2}$ e-mail: info@ahmedvalve.com

Website: www.ahmedvalve.com

Customer Service in USA 800.8르.5륵 